\title{
Rare Presentation of Severely Limited Granulomatosis With Polyangiitis Manifesting With Orbital Wall Destruction: Literature Review and Case Report
}

\author{
Erol HAVUZ1 ${ }^{10}$, Seda GÜDÜL HAVUZ² $\mathbb{B}$ \\ ${ }^{1}$ Department of Ophthalmology, University of Health Sciences, SUAM Samsun Hospital, Samsun, Turkey \\ ${ }^{2}$ Department of Microbiology, Public Health Institution of Turkey, Samsun, Turkey
}

\begin{abstract}
Necrosis resulting from mechanical local factors can be seen in patients with granulomatosis with polyangiitis (GPA) even in remission. GPA can cause serious morbidity even when limited. An ocular prosthesis that increases inflammation and damages local circulation should be used very carefully in such patients. In this article, we report a 68-year-old male patient who was diagnosed with localized GPA 11 years ago and referred to our clinic with the complaint of displacement of an ocular prosthesis inside the nose and epistaxis. Four years ago, the left eye was enucleated because of pain and vision loss. Two months after the enucleation, the patient began to use an ocular prosthesis. Orbital medial wall destruction developed while the patient was receiving maintenance therapy that consisted of cyclophosphamide ( $150 \mathrm{mg} / \mathrm{day})$ plus prednisolone $(32 \mathrm{mg})$. When the ocular prosthesis was displaced in the nasal cavity, the prosthesis was first removed and the patient was clinically stabilized. Later, orbital wall reconstruction was performed at another center.

Keywords: Granulomatosis with polyangiitis, orbital inflammation, ocular prosthesis, orbital wall destruction.
\end{abstract}

Granulomatosis with polyangiitis (GPA), which has also been called Wegener's granulomatosis, is a rare chronic disease coursing with necrotizing granuloma that influences small and medium sized veins. Mechanisms triggering autoimmune inflammation in GPA are not completely known. The disease presents as either the classic generalized form or the limited form. The generalized form affects the lungs, sinuses, and kidneys; the ears, eyes, and nervous system are less influenced. Limited GPA is a form that does not involve vital organs. In limited GPA, granulomatous masses can show invasion, as in tumors in close anatomical areas, and may cause tissue, bone, and cartilage damage. GPA can involve any organ, and the clinical course of the disease can vary greatly depending on the involved organ ${ }^{1-7}$ GPA is a complex disease that causes high morbidity and can even be lethal if not treated. ${ }^{8}$ In limited GPA, granuloma and necrosis areas cause serious morbidity, even when in a limited area, such as the orbital, the pituitary gland, or the nasal cavity. In patients with localized GPA with multiple recurrence, orbital exenteration may be required. . $^{9-11}$

Initial symptoms of GPA can be seen with orbital involvement; ${ }^{6}$ however, orbital involvement is rarely seen in GPA, and only $17 \%$ of granulomatous lesions of the orbital is related to GPA. ${ }^{12}$ However, in some case series, orbital

Received: March 17, 2019 Accepted: May 30, 2019 Published online: February 07, 2020

Correspondence: Erol Havuz, MD. Sağlık Bilimleri Üniversitesi, SUAM Samsun Hastanesi Göz Hastalıkları Kliniği, 55090 İlkadım, Samsun, Türkiye. Tel: +90 505 - 6419995 e-mail: erolhavuz@gmail.com 
involvement in a limited GPA form has been detected in patients with rates as high as $65 \% .{ }^{13}$

In limited GPA, sinonasal involvement, nasal mucosa loss, and moistening problems are seen. Bloody discharge, obstruction, and recurring infections are frequently seen in sinonasal involvement. Erosion of the turbinates, otitis, deafness, saddle nose (which occurs with collapse of the nasal septum), lacrimal duct inflammation, and epiphora from nasal bone erosion are some of the clinical symptoms of localized sinonasal GPA.114 Orbital wall destruction occurring in between $10 \%$ and $69 \%$ of patients has been found in the local form of the diseasee. ${ }^{4,13,15}$

The diagnosis is established with clinical symptoms, cytoplasmic antineutrophil cytoplasmic antibody (c-ANCA) positivity, and histopathological findings. ANCA has been found to be positive in only $65 \%$ of the patients who have GPA with a milder clinical course. ${ }^{14}$ However, the negative c-ANCA test does not exclude GPA diagnosis. The sensitivity of ANCA in GPA diagnosis is 66\%; however, it has been shown to be more significant in identifying disease activity. ${ }^{6}$

To the best of our knowledge, this case report is the first in the literature to show that ocular prosthesis may cause orbital wall necrosis in GPA patients. Therefore, this case report is important and unique.

\section{CASE REPORT}

A 68-year-old male patient was referred to emergency service complaining of displacement of an ocular prosthesis inside the nose and epistaxis. The patient reported general fatigue and weariness that had been continuing for seven days. He reported a diagnosis and regular treatment of localized GPA for 11 years. The initial symptoms and presenting signs of the disease for the described patient were nasal congestion, progressive headaches, and chronic rhinosinusitis; however, we didn't have any data from 11 years ago. The left eye was enucleated four years ago because of pain and vision loss due to necrotizing scleritis and globe perforation caused by vasculitis. Two months after the enucleation, the patient began to use an ocular prosthesis. Four weeks ago, the patient had sinonasal relapse. The patient's loading therapy was a methylprednisolone pulse of $1 \mathrm{~g}$ per day for three days, returned to a $100 \mathrm{mg} /$ day dose, followed by gradual dose reduction to $32 \mathrm{mg} /$ day, and combined cyclophosphamide of $150 \mathrm{mg} /$ day. Vitamin D and calcium was continued on the advice of the internal disease specialist. No pathological symptoms were detected on the patient's chest X-ray, which was taken on the same day. A written informed consent was obtained from the patient.
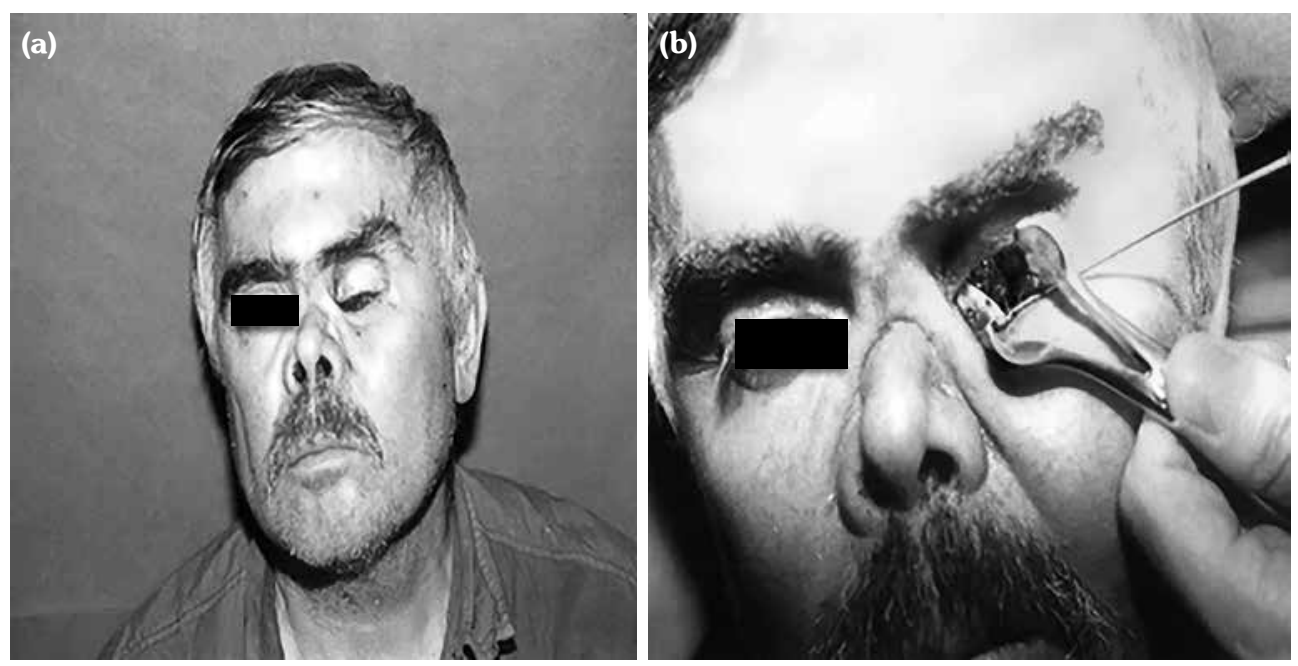

Figure 1. (a) Saddle nose and epistaxis on both sides of nose following granulomatosis with polyangiitis-induced necrosis. (b) Ocular prosthesis inside nasal cavity at orbital medial wall following necrosis. 
The patient's examination revealed collapse of the nasal bridge and epistaxis in both sides of the nose (Figure 1). Ophthalmological examination showed that the left eye was enucleated, there was a $30 \times 20 \mathrm{~mm}$ bone erosion at the left eye orbital medial wall, and the nasal septum was necrotic and destructed. In the right eye, uncorrected visual acuity was 20/20 (Snellen chart). A slit lamp biomicroscopy and fundus examination did not reveal any pathological symptoms. Laboratory test results of the patient were as follows: hemoglobin $13.4 \mathrm{~g} / \mathrm{dL}$, white blood cells $19,500 / \mathrm{mm}^{3}$, and thrombocyte $624,000 / \mathrm{mm}^{3}$. Blood biochemistry results were as follows: blood urea nitrogen $15 \mathrm{mg} / \mathrm{dL}$, creatinine $1 \mathrm{mg} / \mathrm{dL}$, sedimentation $96 \mathrm{~mm} /$ hour, C-reactive protein (CRP) $93 \mathrm{mg} / \mathrm{L}$ with the immunofluorescence antibody method, and c-ANCA was 1/160 titer positive. The patient did not want to undergo radiological tests.

The patient's nasal prosthesis was removed with sedoanalgesia. Topical ciprofloxacin ointment $2 \times 1$ was prescribed. Necrotic hemorrhagic soft tissue and bone pieces of the orbital medial wall were found around the removed prosthesis (Figure 2a). Histopathological examination showed that the multinuclear giant cell was compatible with GPA (Figure 2b), necrotizing vasculitis (Figure 2c), granulomatous inflammation, increased inflammatory cells, and eroded bones (Figure 2d).

With a multidisciplinary approach (cefuroxime, $500 \mathrm{mg}$ twice daily for seven days; prednisolone, $1 \mathrm{mg} / \mathrm{kg} /$ day and once every four weeks; and cyclophosphamide therapy, 1,000 mg single dose intravenous pulse) was continued for six months with a rheumatologist and otorhinolaryngologist. Clinical and laboratory remission were ensured in the third month. Three months after the beginning of systemic treatment, inflammatory markers, such as sedimentation and CRP, returned to normal. Since ANCA titer is not a predictive marker for limited GPA patients, it was not tested during the remission period. Three months after remission, a bone graft was grafted to the orbital medial wall in another tertiary center.

\section{DISCUSSION}

PubMed and Web of Science were searched using the keywords, "Wegener's granulomatosis," "Granulomatosis with polyangiitis," "orbital wall destruction," "orbital wall necrosis," and "orbital bone erosion." The search strategy for PubMed and Web of Science is shown in Appendix 1. Of the resulting articles, five were from PubMed
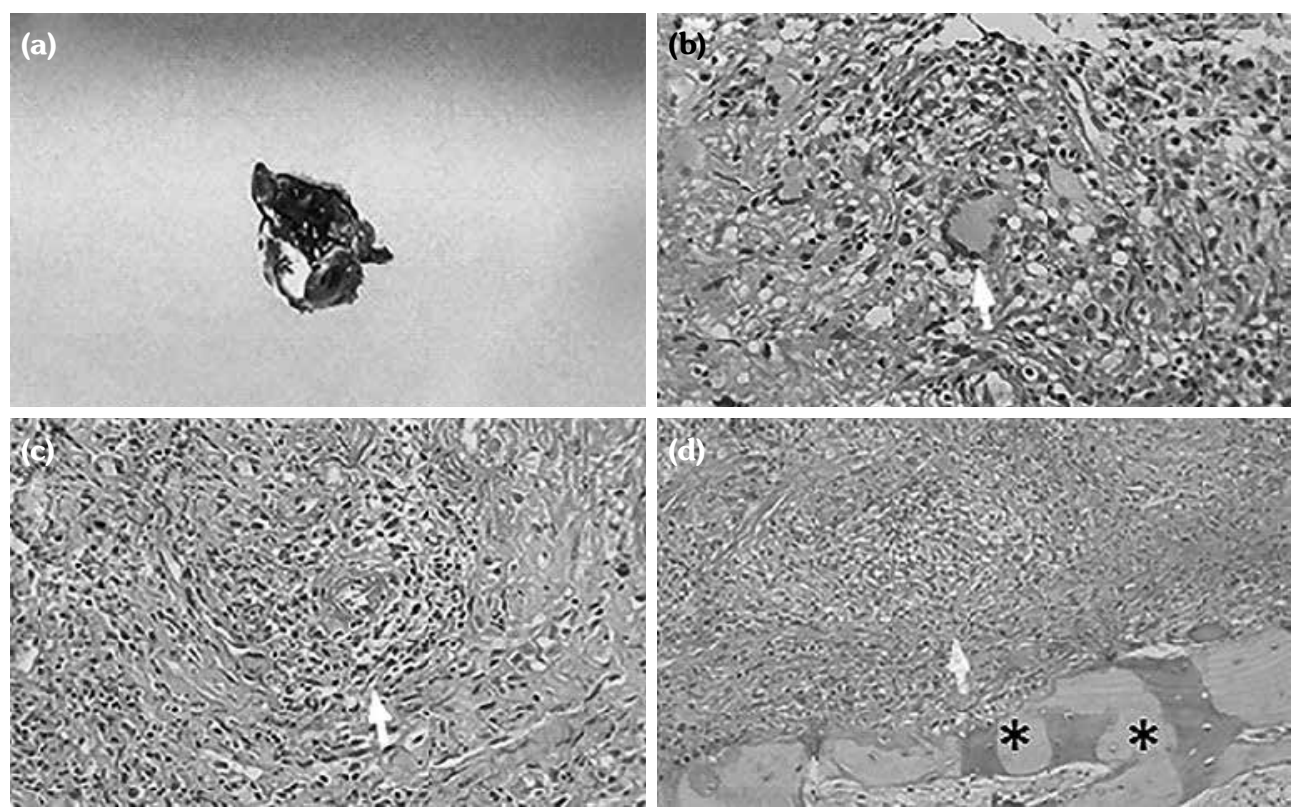

Figure 2. (a) Necrotic hemorrhagic soft tissue and bone pieces adherent on ocular prosthesis. (b) Multinucleated giant cell, $(\mathrm{H}-\mathrm{E} \times 100)$. (c) Vasculitis, $(\mathrm{H}-\mathrm{E} \times 50)$. (d) Acute and chronic cell infiltration, granulomatous inflammation $(\mathrm{H}-\mathrm{E} \times 20)$. 


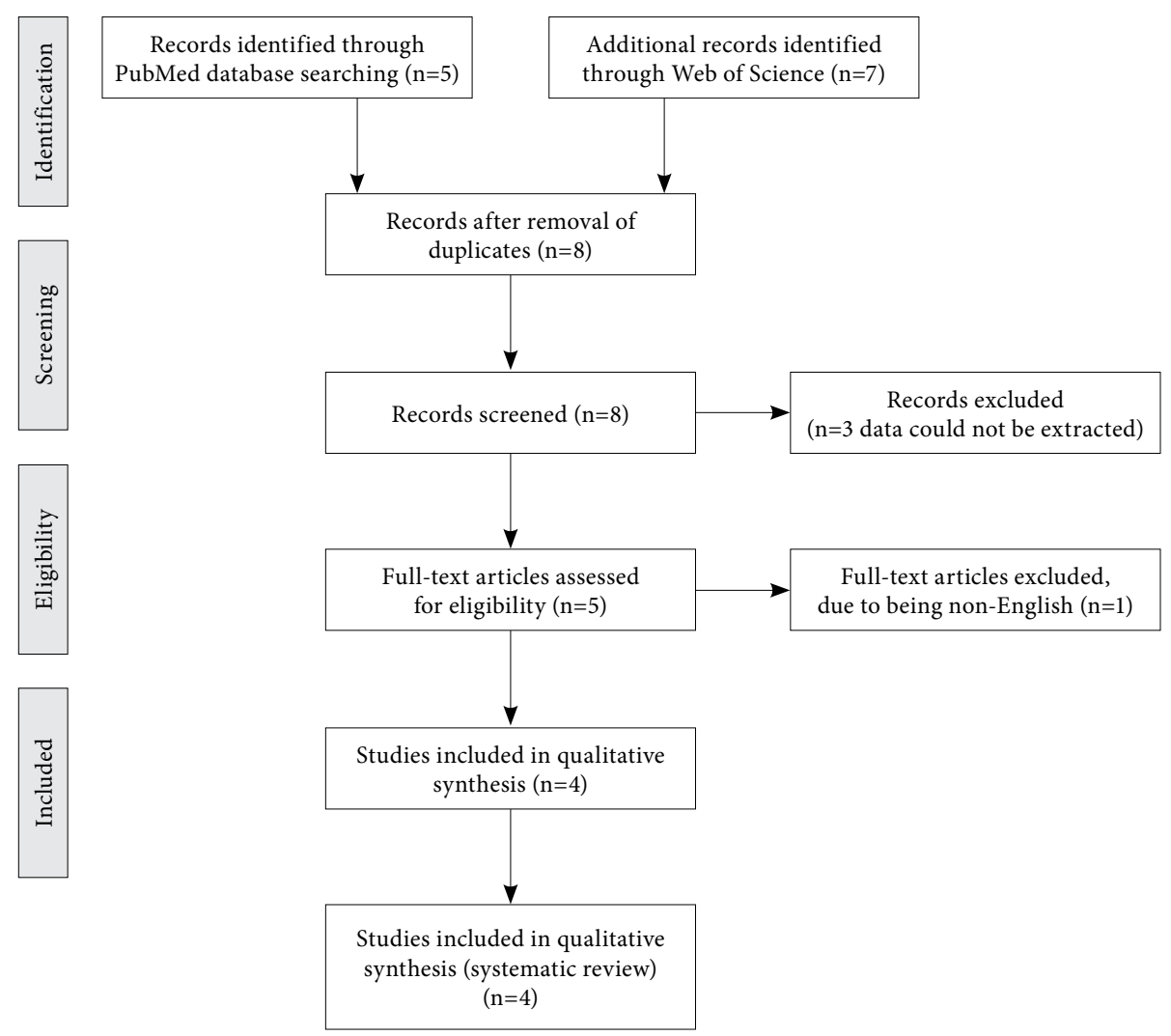

Figure 3. Flow diagram posing selection of studies and screening.

and seven were from the Web of Science. The articles were chosen in line with the flow diagram shown in Figure 3. All case reports, abstracts, letters to the editors, and original articles were included. Three articles were excluded according to the different bone necrosis, such as ethmoid, sphenoid, and posterior septum. No article was detected when the keywords "ocular prosthesis" and "nasal cavity" were searched together.

Of the studies obtained, 221 patients with localized GPA were noted. Average ages of these patients varied between 43 and 53 years. When the studies were reviewed, the most commonly found symptoms were orbital mass and proptosis, which varied between $24 \%$ and $69 \%$. The literature reviews are shown in Table 1.

Symptoms were seen with a broad clinical spectrum, such as epiphora, orbital mass, swelling of the lids, diplopia, episcleritis, ulcerative keratitis, lacrimal system obstruction, conjunctival mass, lacrimal mass, compressive neuropathy, ischemic neuropathy, anteroposterior uveitis, pain, and vision loss. The least found symptoms were ischemic neuropathy and posterior uveitis, with a rate of $1.8 \%$. In all of the studies, in addition to orbital involvement, at least one symptom related to sinonasal involvement was noted, which was not life-threatening but which had high morbidity and was diagnosed as saddle nose, septal perforation, bony erosion, otitis media, and/or subglottic stenosis. In the studies of Holle et al. ${ }^{4}$ and Tan et al., ${ }^{16}$ nasal bone erosions were found at a rate of $18 \%$ and $32 \%$, respectively. In terms of orbital wall destruction, although the rate of occurrence was not given in Tan et al.'s study, ${ }^{16}$ the rate was $10 \%$ in Holle et al.'s study, ${ }^{4} 13.2 \%$ in Ismailova et al.'s study, ${ }^{17}$ and $21 \%$ in Woo et al.'s study. ${ }^{13}$

In all studies except Ismailova et al.'s, ${ }^{17}$ ANCA positivity rates were found to be close, and 35\% to $47 \%$ of the cases were found to be positive. Ismailova et al. ${ }^{17}$ grouped 74 patients with orbital involvement into groups of patients (1) with orbital mass without lacrimal gland involvement, (2) with lacrimal gland involvement, and (3) with extraocular myositis. ANCA positivity was found 


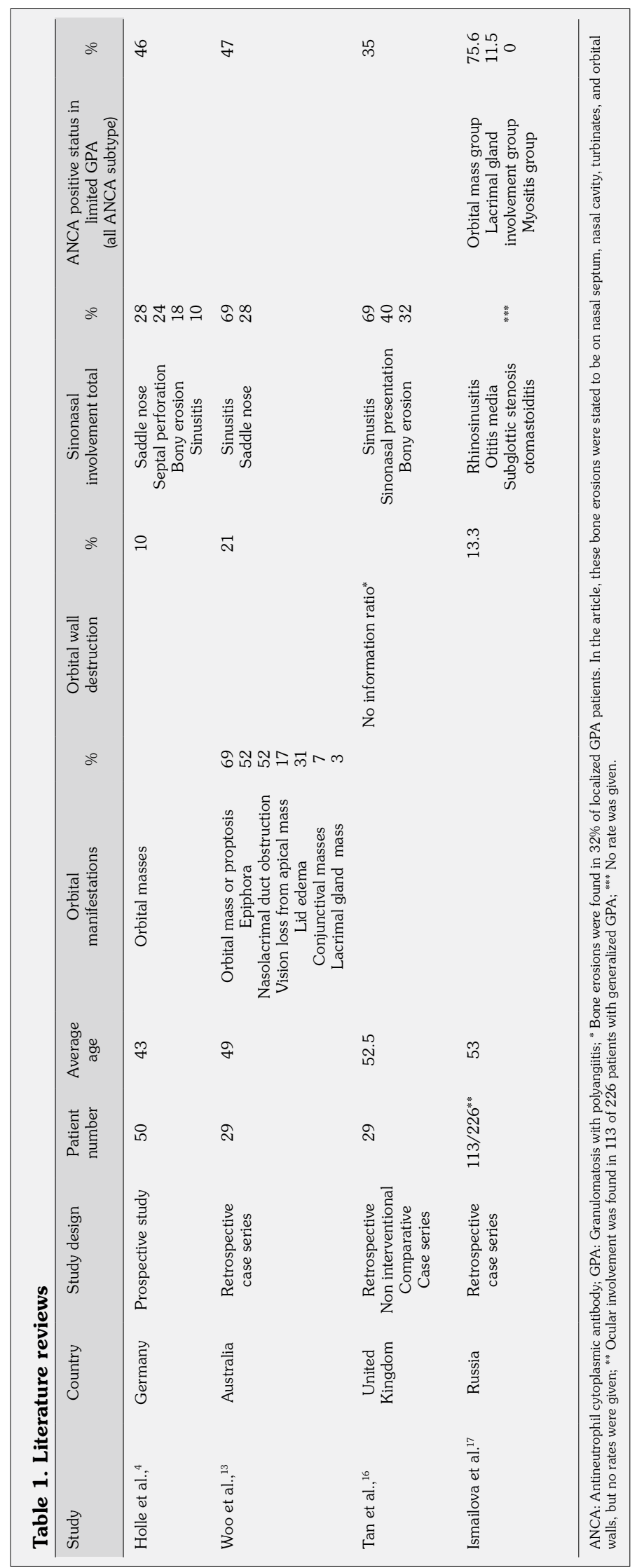


to be $75.6 \%, 11.5 \%$, and $0 \%$, respectively, in these patients.

A pathological classic triad of the disease is vasculitis, tissue necrosis, and granulomatous inflammation. Damage in ocular structures may result from vasculitis and nutritional disorder, spread of orbital cellulites, pressure of granulomatous mass, ${ }^{8}$ and acute or chronic inflammation. ${ }^{3}$ Ocular manifestation of the disease occurs as a result of either neighboring granulomatous sinusitis or focal vasculitis. In reviewed articles, orbital mass and proptosis were the most frequent orbital presentations in the studies of Holle et al., ${ }^{4}$ Tan et al., ${ }^{16}$ Ismailova et al., ${ }^{17}$ and Woo et al., ${ }^{13}$ they occurred at rates of $24 \%, 48 \%, 65.4 \%$, and $69 \%$, respectively. Studies have indicated high visual losses, as much as $57.9 \% .^{18}$ In one patient, although there was no granulomatous mass in the orbital, intense inflammation caused by necrosis in bone tissues was found to cause bilateral optic neuritis development. ${ }^{10}$ The diffuse disease in the orbital may cause bone destruction or a new bone formation. ${ }^{15,19}$

In the study by Fechner et al., ${ }^{11}$ which consisted of 15 patients with GPA, only orbital involvement was found in $80 \%$ of the cases and sinonasal involvement was found in $20 \%$ of the cases. However, Wojciechowski et al. ${ }^{20}$ stated that 80 to $95 \%$ of the initial symptoms of GPA patients were otorhinolaryngology manifestations.

In the reviewed articles, although sinusitis was found to occur at a rate of 10 to $69 \%$, complications such as septum perforation and saddle nose were found to occur at varying rates. In patients with GPA, if there is nasal or sinonasal involvement, bone erosions may also be expected. When patients with sinonasal GPA were computed tomography-scanned, erosion was found in orbital bones at a rate of $20 \% .^{1}$ In patients with GPA, bone damage can occur as a result of acute or chronic cellular infiltration of the vein wall, fibrinoid degeneration in micro-abscess and collagen, and the formation of great granulomas and the pressure they have on neighboring small arteries. ${ }^{11,21,22}$ The fact that destruction is seen not only in orbital walls close to orbital mass but also in the nasal septum and nose cartilages supports the view that the mass has local inflammatory effects rather than direct effects. ${ }^{23}$
In our case report, granulomatous and necrotic tissue were detected around the prosthesis removed from the nasal cavity. The patient had been receiving maintenance therapy consisting of cyclophosphamide and glucocorticoid. However, the complaints of fatigue and weariness that began within the last week can be associated to disease progression. During this period, the patient was not referred to a rheumatology clinic. It was thought that the wall destruction in this patient is associated with both local inflammatory events and the inflammation increasing effect of the prosthesis.

Cytoplasmic ANCA patterns can have up to 95-98\% specificity for GPA, and it increases in the acute phase. ${ }^{18}$ In the studies reviewed, ANCA positivity differed between $11.5 \%$ and $75.6 \%$. ANCA positivity rate and wall destruction do not seem to be correlated. In patients with limited GPA, ANCA positivity rates differed. In Isa et al.'s study, ${ }^{24}$ only 12 of 36 limited GPA patients with ocular involvement were found to have ANCA positivity.

In patients with GPA with orbital involvement, progression can be seen despite remission treatment with cyclophosphamide and glucocorticoid. Holle et al. ${ }^{23}$ showed that $41 \%$ of the patients did not respond sufficiently to this dual treatment. In the reviewed articles, the treatments received by patients with orbital wall destruction were not specified. For this reason, it is unknown which patients receiving which treatment were inclined to develop wall destruction.

Moreover, allergic immune responses associated with ocular prosthesis can be seen. Although this immune response may result from the surface material of the prosthesis, it can also be associated with the debris accumulated on the prosthesis. ${ }^{25,26}$ The fact that cases developed such a rare complication despite maintenance therapy was thought to be associated with the prosthesis and active disease. Not only ocular prosthesis but also local inflammatory events induced by prosthesis and active disease were thought to have caused necrosis. There was no direct mechanical effect, such as blind trauma or friction in the ocular history.

Nasal deformities associated with GPA disease are usually not treated. Surgical 
approaches are not recommended because of the tendency for infections, poor tissue perfusion, and wound healing. Surgery should be performed only on patients in remission and by choosing the correct technique. ${ }^{20}$ Orbital wall reconstruction was performed on our patient whose ocular prosthesis was displaced in the nasal cavity after he was clinically stabilized. In patients with a high vasculitis damage index, which is the indicator of chronic disease, orbital wall destruction develops during the follow-up period. ${ }^{23}$

In conclusion, orbital bones should be followed-up closely in terms of destruction in chronic patients even if they are receiving maintenance therapy. It should be kept in mind that both autoimmunity and prosthesis-induced inflammation in GPA may lead to orbital wall necrosis.

\section{Declaration of conflicting interests}

The authors declared no conflicts of interest with respect to the authorship and/or publication of this article.

\section{Funding}

The authors received no financial support for the research and/or authorship of this article.

\section{REFERENCES}

1. Zycinska K, Wardyn KA, Piotrowska E, Zielonka TM, Zycinski $\mathrm{H}$, Bogaczewicz A, et al. Rhinologic and sinonasal changes in PR3 ANCA pulmonary vasculitis. Eur J Med Res 2010;15:241-3.

2. Leavitt RY, Fauci AS, Bloch DA, Michel BA, Hunder GG, Arend WP, et al. The American College of Rheumatology 1990 criteria for the classification of Wegener's granulomatosis. Arthritis Rheum 1990;33:1101-7.

3. Muller K, Lin JH. Orbital granulomatosis with polyangiitis (Wegener granulomatosis): clinical and pathologic findings. Arch Pathol Lab Med 2014;138:1110-4.

4. Holle JU, Gross WL, Holl-Ulrich K, Ambrosch P, Noelle B, Both M, et al. Prospective long-term followup of patients with localised Wegener's granulomatosis: does it occur as persistent disease stage? Ann Rheum Dis 2010;69:1934-9.

5. Yamasaki S, Eguchi K, Kawabe Y, Tsukada T, Nagataki S. Wegener's granulomatosis overlapped with Takayasu arteritis. Clin Rheumatol 1996;15:303-6.
6. Santiago YM, Fay A. Wegener's granulomatosis of the orbit: a review of clinical features and updates in diagnosis and treatment. Semin Ophthalmol 2011;26:349-55.

7. Pakalniskis MG, Berg AD, Policeni BA, Gentry LR, Sato Y, Moritani T, et al. The Many Faces of Granulomatosis With Polyangiitis: A Review of the Head and Neck Imaging Manifestations. AJR Am J Roentgenol 2015;205:W619-29.

8. Kubaisi B, Abu Samra K, Foster CS. Granulomatosis with polyangiitis (Wegener's disease): An updated review of ocular disease manifestations. Intractable Rare Dis Res 2016;5:61-9.

9. Baird SM, Pratap U, McLean C, Law CP, Maartens N. Rare presentation of Wegener's granulomatosis in the pituitary gland: Case report and literature review. Int J Surg Case Rep 2017;33:24-6.

10. Monteiro ML, Borges WI, do Val Ferreira Ramos C, Lucato LT, Leite CC. Bilateral optic neuritis in wegener granulomatosis. J Neuroophthalmol 2005;25:25-8.

11. Fechner FP, Faquin WC, Pilch BZ. Wegener's granulomatosis of the orbit: a clinicopathological study of 15 patients. Laryngoscope 2002;112:1945-50.

12. Satorre J, Antle CM, O'Sullivan R, White VA, Nugent RA, Rootman J. Orbital lesions with granulomatous inflammation. Can J Ophthalmol 1991;26:174-95.

13. Woo TL, Francis IC, Wilcsek GA, Coroneo MT, McNab AA, Sullivan TJ; Australasian Orbital and Adnexal Wagener's Study Group. Australasian orbital and adnexal Wegener's granulomatosis. Ophthalmology 2001;108:1535-43.

14. Tarabishy AB, Schulte M, Papaliodis GN, Hoffman GS. Wegener's granulomatosis: clinical manifestations, differential diagnosis, and management of ocular and systemic disease. Surv Ophthalmol 2010;55:429-44.

15. Provenzale JM, Mukherji S, Allen NB, Castillo $\mathrm{M}$, Weber AW. Orbital involvement by Wegener's granulomatosis: imaging findings. AJR Am J Roentgenol 1996;166:929-34.

16. Tan LT, Davagnanam I, Isa H, Taylor SR, Rose $\mathrm{GE}$, Verity $\mathrm{DH}$, et al. Clinical and imaging features predictive of orbital granulomatosis with polyangiitis and the risk of systemic involvement. Ophthalmology 2014;121:1304-9.

17. Ismailova DS, Abramova JV, Novikov PI, Grusha YO. Clinical features of different orbital manifestations of granulomatosis with polyangiitis. Graefes Arch Clin Exp Ophthalmol 2018;256:1751-6.

18. Jiang B, Zhao YY, Wei SH. Granulomatosis with polyangiitis: the relationship between ocular and nasal disease. Ocul Immunol Inflamm 2013;21:115-8.

19. Fishman JM, Slovick A, East CA. Wegener's granulomatosis of the orbit: two cases requiring endoscopic surgical decompression. J Laryngol Otol 2008;122:1257-9.

20. Wojciechowska J, Krajewski W, Krajewski P, Krecicki T. Granulomatosis with polyangiitis in otolaryngologist 
practice: A review of current knowledge. Clin Exp Otorhinolaryngol 2016;9:8-13.

21. Ostri C, Heegaard S, Prause JU. Sclerosing Wegener's granulomatosis in the orbit. Acta Ophthalmol 2008;86:917-20.

22. Lloyd G, Lund VJ, Beale T, Howard D. Rhinologic changes in Wegener's granulomatosis. J Laryngol Otol 2002;116:565-9.

23. Holle JU, Voigt C, Both M, Holl-Ulrich K, Nölle B, Laudien $\mathrm{M}$, et al. Orbital masses in granulomatosis with polyangiitis are associated with a refractory course and a high burden of local damage. Rheumatology (Oxford) 2013;52:875-82.
24. Isa H, Lightman S, Luthert PJ, Rose GE, Verity DH, Taylor SR. Histopathological features predictive of a clinical diagnosis of ophthalmic granulomatosis with polyangiitis (GPA). Int $J$ Clin Exp Pathol 2012;5:684-9.

25. Litwin AS, Worrell E, Roos JCP, Edwards B, Malhotra R. Can We improve the tolerance of an ocular prosthesis by enhancing its surface finish? Ophthalmic Plast Reconstr Surg 2018;34:130-5.

26. Akman A, Irkeç M, Orhan M, Erdener U. Effect of lodoxamide on tear leukotriene levels in giant papillary conjunctivitis associated with ocular prosthesis. Ocul Immunol Inflamm 1998;6:179-84.

\section{Appendix 1.}

\section{Search strategy for Web of Science:}

Set 1 Ts= (Wegener * Granulomatosis AND [orbita* wall destruction OR orbita* wall necrosis OR orbita* bone erosion])

Set 2 TS = ([Granulomatosis] [of] [polyangiitis] AND [orbita*] [wall] [destruction] OR [orbita*] [wall] [necrosis] OR [orbita*] [bone) [erosion])

Set $3 \quad 1$ OR 2

No restrictions were employed such languages, document types, timespan and Web of Science Core Collection.

\section{Search strategy for PubMed:}

1 "granulomatosis with polyangiitis"[MeSH Terms] OR (“granulomatosis"[All Fields] AND "polyangiitis”[All Fields]) OR "granulomatosis with polyangiitis"[All Fields])

2 "wegener"[All Fields] AND "granulomatosis"[All Fields]) OR "wegener's granulomatosis"[All Fields])

3 "(orbita"[All Fields] AND wall[All Fields] AND destruction[All Fields])

4 "(orbita*[All Fields] AND wall[All Fields] AND ("necrosis"[MeSH Terms] OR "necrosis"[All Fields]))

5 “(orbital”[All Fields] AND "bone"[All Fields]) OR "orbital bone"[All Fields]) AND erosion[All Fields]))

$6 \quad 3$ OR 4 OR 5

$7 \quad 1$ AND 6

$8 \quad 2$ AND 6 